based immunocytochemistry assays on SH-SY5Y (human neuroblastoma) cell cultures. The association between serum positivity for $\mathrm{AnAb}$ by IHC and a large panel of data (demographic, serologic, SLEDAI, conventional brain MRI, treatment) was investigated by univariate analysis. Multivariate models were fitted with covariates with $\mathrm{p}<0.05$ to identify factors independently associated with serum positivity for AnAb; $p<0.05$ were considered statistically significant.

Results AnAb were detected in 23 (82.1\%) NPSLE patients and in $16(39.0 \%)$ SLE patients without NP involvement resulting in $82 \%$ specificity (95\% CI $71 \%-90 \%)$ and $61 \%$ sensitivity (95\%CI 48\%-72\%) in differentiating NPSLE from SLE without NP involvement. None of the sera from MS patients $(0 \%)$ and healthy subjects (0\%) showed AnAb. Serum AnAb by IHC were independently associated with NPSLE $(p<0.01)$ and higher SLEDAI $(\mathrm{p}<0.01)$. No association with specific NPSLE syndrome and brain conventional MRI abnormalities was identified.

Conclusion $\mathrm{AnAb}$ are significantly more frequent in patients with NPSLE than SLE. Further studies are needed to identify the unknown neuronal antigens targeted by AnAb in SLE patients.

\section{P18 PRESENCE OF RHEUMATOID FACTOR WAS ASSOCIATED WITH A DECREASED RISK OF LUPUS NEPHRITIS IN PATIENTS WITH JUVENILE SYSTEMIC LUPUS ERYTHEMATOSUS} ${ }^{1}$ Charlene Foley, ${ }^{1}$ Coziana Ciurtin. ${ }^{1}$ Centre for Adolescent Rheumatology, University College London, London; ' University College London Medical School, London, UK

\subsection{6/lupus-2020-eurolupus.67}

Background Estimated 10- 20\% of all patients with systemic lupus erythematosus (SLE) develop clinical disease before the age of 18 years and are therefore classified as juvenile-onset SLE (JSLE). JSLE is characterised by a higher prevalence of lupus nephritis, compared to adult- onset SLE. Chronic kidney disease (CKD) refers to a state of irreversible kidney damage and/or reduction of kidney function that is associated with progressive loss of function over time. Lupus nephritis does not always lead to CKD. However, when it does it is associated with increased morbidity and mortality.

Objectives We aimed to identify clinical and laboratory predictors of CKD development in JSLE patients by comparing the baseline characteristics of JSLE patients with and without CKD to ascertain if there are any significant differences between the two groups.

Methods This is a single-centre retrospective study, who included patients reviewed in our young adult and adolescent clinics. Mann- Whitney U or Chi- square test were performed to compare the characteristics between the patients with and without CKD. We used the Pearson's ( $r$ ) or Kendall's $\tau$ (tau) correlation to examine if there is any association between the CKD and the baseline characteristics.

Results We identified 44 JSLE patients, out of which 17 (39\%) fulfilled the diagnostic criteria for CKD at their last clinical review. The stages of CKD varied from 2 to 5. All patients with CKD also had lupus nephritis, while 5/44 patients (11\%) had lupus nephritis without CKD. The baseline characteristics are detailed in the table 1 below. There were statistically significant differences in the treatments used for patients with and without CKD. As expected, the highest
Abstract P18 Table 1 Baseline characteristics of JSLE patients in the Arthritis UK Centre for Adolescent Rheumatology

\begin{tabular}{llll}
\hline & CKD & Without CKD & P \\
\hline Number of patients, n (\%) & $17(39 \%)$ & $27(61 \%)$ & \\
Female, n (\%) & $14(80 \%)$ & $23(85.2 \%)$ & $\mathrm{p}=0.47$ \\
Age at diagnosis, years & $12(10-11)$ & $12(11-15)$ & $\mathrm{p}=0.62$ \\
Disease duration, years & $12(8-14)$ & $12(7-13)$ & $\mathrm{p}=0.78$ \\
Highest dsDNA & $215(34-644)$ & $32(6.8-104)$ & $\mathrm{p}=0.03^{*}$ \\
SLEDAI at last assessment & $2(0-4)$ & $0(0-2)$ & $\mathrm{p}=0.33$ \\
Arthritis, n (\%) & $13(76 \%)$ & $16(59 \%)$ & $\mathrm{p}=0.24$ \\
Rituximab, number of courses & $1.5(0-2.3)$ & $0(0-1)$ & $\mathrm{p}=0.04^{*}$ \\
Mycophenolate Mofetil, months & $44(21-96)$ & $17(0-70)$ & $\mathrm{p}=0.04^{*}$ \\
Steroids, months & $48(37-82)$ & $25(11-50)$ & $\mathrm{p}=0.03^{*}$ \\
\hline
\end{tabular}

Numbers are medians (interquartile ranges) unless otherwise stated.

${ }^{*} p<0.05$ is significant

dsDNA levels were higher in patients with CKD $(\mathrm{p}=0.03)$. There was also a positive moderate correlation $(\rho=0.32)$ between raised levels of dsDNA and the development of CKD $(\mathrm{p}=0.008)$. We also found a negative moderate correlation $(\tau=-0.439)$ between the presence of RF and CKD $(p=0.04)$.

Conclusion Acknowledging the limitations posed by this small study, we identified a negative moderate correlation between the presence of $\mathrm{RF}$ and $\mathrm{CKD}$, which has also been reported in the literature before. We cannot conclude that RF exerts a protective effect against renal disease in SLE, because of the many confounders that might account for a decreased RF in JSLE. Further research using a large JSLE cohort enabling multivariate logistic regression is recommended. DsDNA antibody levels are a measure of disease activity in lupus nephritis and therefore this might explain why patients who developed CKD were noted to have higher anti-dsDNA levels, in comparison with the patients who did not develop CKD.

\section{P19 AUTOANTIBODY PROFILE ANALYSIS IN SLE PATIENTS}

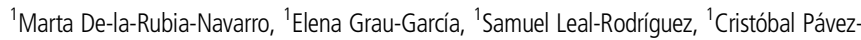
Perales, ${ }^{1}$ Cristóbal Alcañiz-Escandell, ${ }^{1}$ Inés Cánovas-OImos, Inmaculada Chalmeta-Verdejo, ${ }^{1} J o r g e ~ J u a n$ Fragio-Gil, ${ }^{1}$ Roxana González-Mazarí, ${ }^{1}$ Luis González-Puig, 'José Ivorra-Cortés, ${ }^{1}$ Isabel Martínez-Cordellat, ${ }^{1}$ Carmen Nájera-Herranz, ${ }^{1}$ Rosa Negueroles-Albuixech, ${ }^{1} J o s e ́$ Eloy Oller-Rodríguez, ${ }^{1}$ Francisco Miguel Ortiz-Sanjuán, ${ }^{1}$ Elvira Vicens-Bernabeu, ${ }^{2}$ Daniel Hervás-Marín, ${ }^{3}$ Meritxel Fernández Matilla, ${ }^{3}$ Nagore Fernández-Llanio Comella, ${ }^{3} J u a n$ Antonio Castellano Cuesta, ${ }^{1} J o s e ́$ Andrés Román-Ivorra. ${ }^{1}$ Rheumatology Dept., HUPLa-Fe, Valencia; ${ }^{2}$ Biostatistics Unit, IIS-La-Fe, Valencia; ${ }^{3}$ Rheumatology Section, HospitalArnau-de-Vilanova, Valencia, Spain

\subsection{6/lupus-2020-eurolupus.68}

Background/Purpose In Systemic Lupus Erythematosus (SLE) the presence of some autoantibodies is related to specific clinical manifestations. We aimed to define SLE patient groups according to an autoantibody profile and to analyze the correlation of these profiles to clinical manifestations.

Methods A cross-sectional observational study of SLE (SLICC 2012 criteria) was conducted. A clinical and analytical evaluation was performed. Clinical manifestations were described according to RELESSER study.

We selected 8 autoantibodies to classify SLE patients: antidsDNA, anti-Sm, anti-RNP, anticardiolipin IgG/IgM (aCL IgG/ $\mathrm{M})$, anti-ß2microglobulin $\operatorname{IgG} / \operatorname{IgM}(\mathrm{a} 2 \mathrm{M} \operatorname{IgG} / \mathrm{M})$, lupus anticoagulant (LA), anti-Ro and anti-La. Immunological profiles 
were created according to previous study of Artim-Esen B et al. 2014.

Results 142 SLE patients with a mean age at diagnosis of 33.29 (13.53) and a mean time of disease evolution of 15.82 (10.56) years were evaluated. Mean SLEDAI score was 5.91 (5.6) and mean SLICC value 1.1 (1.46). Autoantibody frequencies are: ANAs 87.3\%, anti-dsDNA 36.62\%, anti-Sm 9.2\%, anti-RNP 3.5\%, aCL IgG/M 20.15\%, aß2M IgG/M 21.88\%, LA 26.27\%, anti-Ro $45.07 \%$ and anti-La 16.2\%.

Profile $n^{\circ} 2$ included SLE patients with anti-Sm/RNP positivity. Profile $\mathrm{n}^{\circ} 3$ included patients with anti-Ro/La positivity. Profile $\mathrm{n}^{\circ} 4$ included patients with aCL IgG/M or $\mathrm{aB} 2 \mathrm{M}$ IgG/ $\mathrm{M}$ or LA positivity. Profile $\mathrm{n}^{\circ} 5$ included patients who exclusively showed anti-DNA positivity. Profile $n^{\circ} 1$ included all patients excluded from the other profiles.

Profile $\mathrm{n}^{\circ} 1$ patients were diagnosed earlier and had a longer disease evolution, whereas Profile $n^{\circ} 5$ patients were diagnosed later and with shorter disease evolution.

We observed a significant association among hematological affection and high levels of anti-Ro $(\mathrm{P}<0.0001)$, anti-La $(\mathrm{P}=0.022)$ and anti-Sm $(\mathrm{P}=0.018)$ was observed.

Conclusions An association between the presence of anti-Ro/La and hematological affection, as well as high incidence of Sjögren syndrome in these subgroup of patients was described.

\section{P20 ABSTRACT WITHDRAWN}

\section{\begin{tabular}{|l|l}
\hline P21 INVESTIGATION OF POSSIBLE PATHOGENIC \\
\hline
\end{tabular} AUTOANTIBODIES IN MEMBRANOUS LUPUS NEPHRITIS}

${ }^{1}$ Filipa Farinha, ${ }^{2}$ Ruth J Pepper, ${ }^{1}$ Chris Wincup, ${ }^{2}$ Alan Salama, ${ }^{1}$ David A Isenberg, ${ }^{1}$ Anisur Rahman. ${ }^{1}$ Centre for Rheumatology, University College London, London; ${ }^{2}$ Centre for Nephrology, University College London-Royal Free Campus, London, UK

10.1136/lupus-2020-eurolupus.69
Background The pathogenesis of membranous lupus nephritis (MLN) is poorly understood. Our objectives were to investigate, in patients with MLN, the presence of antibodies to nucleosomes and histones, which have been linked to the pathogenesis of proliferative lupus nephritis (PLN); and antibodies against phospholipase A2 receptor (PLA2R), previously identified in patients with idiopathic membranous nephropathy (IMN).

Methods Single-centre study, using banked serum samples from patients with biopsy-proven lupus nephritis (LN). For each patient, we aimed to identify two paired serum samples - one at the date of renal biopsy (active nephritis) and one when LN was quiescent. Antibodies were detected with indirect ELISA. Positivity cut-offs were $20 \mathrm{U} / \mathrm{mL}$ for anti-nucleosomes and PLA2R, and $40 \mathrm{U} / \mathrm{mL}$ for anti-histones antibodies. Levels of antibodies in paired active and quiescent LN samples were compared with a Wilcoxon signed-rank test, and a KruskalWallis test was used to compare the levels between independent LN groups.

Results Forty-nine active serum samples (13 pure MLN, 22 PLN and 14 mixed) and 41 paired quiescent samples were tested for anti-nucleosome and anti-PLA2R antibodies; and 44 active (13 pure MLN, 17 PLN and 14 mixed) and 38 paired quiescent samples were tested for anti-histones antibodies. Anti-nucleosome antibodies were present in $98 \%$ of active-LN patients. Total median level was 190.70 (IQR 681), with no significant difference between the three groups $(p=0.730)$. During quiescent $L N$, levels dropped significantly in PLN but not in pure MLN patients (figure 1). Anti-histone antibodies were positive in $32 \%$ of active- $\mathrm{LN}$ patients, with no significant difference between groups $(\mathrm{p}=0.828)$; their levels showed a similar trend to that of anti-nucleosomes (figure 1). Antibodies to PLA2R were negative in all patients.

Conclusions Anti-nucleosome and anti-histone antibody levels did not reflect disease activity in MLN. Anti-PLA2R antibodies were absent in these patients. None of these antibodies is likely to be playing a preponderant role in MLN pathogenesis.
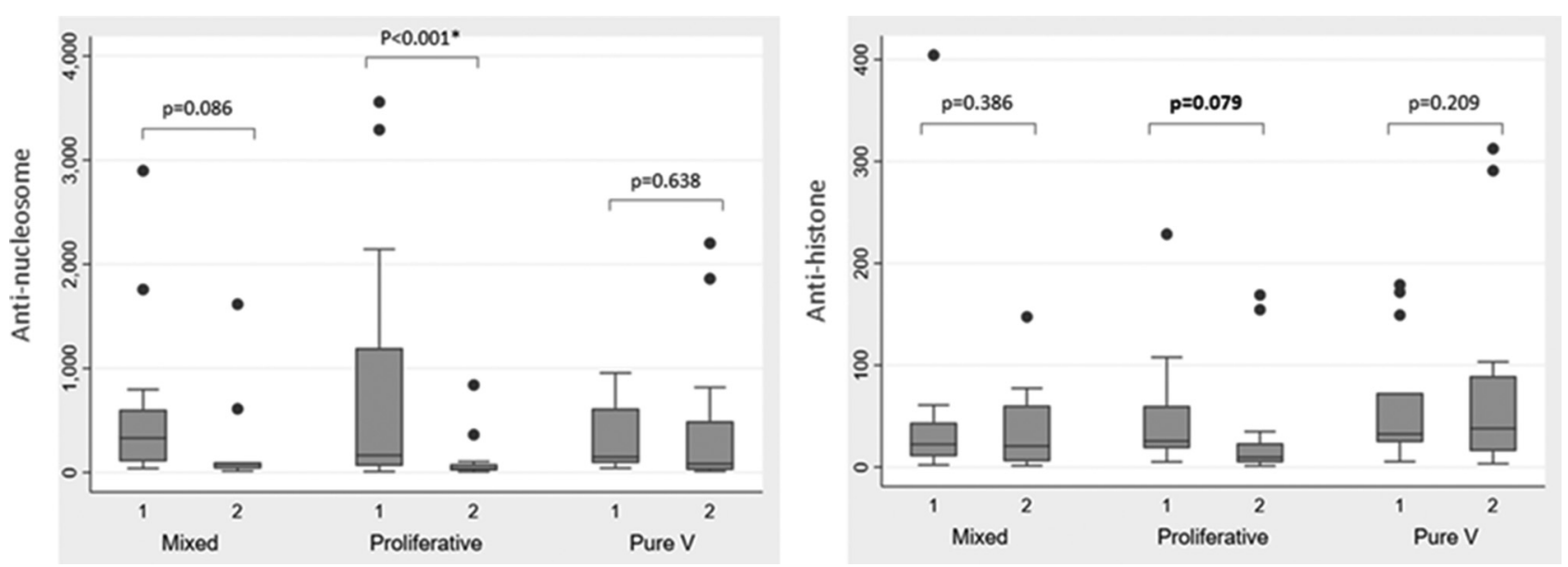

Abstract P21 Figure 1 Anti-nucleosome and anti-histone antibody levels (U/mL) in patients with mixed, proliferative and membranous $L N$, when the nephritis was active (1) and quiescent (2) 